\title{
Cavitation and Flow Instabilities in a 3- Bladed Axial Inducer Designed by Means of a Reduced Order Analytical Model
}

\author{
Angelo Cervone \\ Project Manager - Alta S.p.A. \\ Ospedaletto - Pisa, Italy \\ a.cervone@alta-space.com
}

\author{
Angelo Pasini \\ Ph.D. Student, Aerospace Engineering Department \\ Pisa University, Italy \\ a.pasini@alta-space.com
}

\author{
Lucio Torre \\ Project Manager - Alta S.p.A. \\ Ospedaletto - Pisa, Italy \\ l.torre@alta-space.com
}

\author{
Luca d'Agostino \\ Professor, Aerospace Engineering Department \\ Pisa University, Italy \\ luca.dagostino@ing.unipi.it
}

\begin{abstract}
The present paper illustrates the main results of an experimental campaign conducted using the CPRTF (Cavitating Pump Rotordynamic Test Facility) at Alta S.p.A. The tests were carried out on the DAPAMITO inducer, a three-bladed axial pump designed and manufactured by Alta S.p.A. using a simplified analytical model for the prediction of geometry and noncavitating performance of typical space rocket inducers. The transparent inlet section of the facility was instrumented with several piezoelectric pressure transducers located at three axial stations: inducer inlet, outlet and the middle of the axial chord of the blades. At each axial station at least two transducers were mounted with given angular spacing in order to cross-correlate their signals for amplitude, phase and coherence analysis. However, probably because of the high value of the blade tip clearance, very few flow instabilities have been detected on the inducer, including: steady asymmetric cavitation caused by the different extension of the cavitating regions on the blades; cavitation surge at a frequency equal to 0.16 times the inducer rotational frequency; a higher-order axial phenomenon at 7.2 times the rotational frequency.
\end{abstract}

\section{INTRODUCTION}

Space rockets turbopumps are one of the most crucial components of all primary propulsion concepts powered by liquid propellant engines. The design of high power density, dynamically stable machines capable of meeting the extremely demanding pumping, suction and reliability requirements of space transportation systems is driven by significant limitations and constraints (Stripling \& Acosta [1]).
Usually, rocket propellant feed turbopumps employ an inducer upstream of the centrifugal stage. The main purpose of the inducer is to pressurize the flow sufficiently for enabling the main pump to operate satisfactorily, in order to avoid unacceptable cavitation, improve the suction performance and reduce the propellant tank pressure and weight. Typical space rocket inducers are characterized by fewer blades than centrifugal pump impellers, lower design flow coefficients, large stagger angles and significantly high blade solidities. Long blades and small incidence angles allow for a sufficiently high residence time for the collapse of cavitation bubbles and the gradual exchange of energy with the flow. This results in a beneficial configuration from the point of view of cavitating performance, which in turn results in highly viscous, turbulent and nonisentropic flow inside the blade channels.

The occurrence of cavitation makes the inducer one of the most critical components of the turbopump assembly as a consequence of the possible development of flow instabilities that can seriously degrade the performance of the machine, or even cause its rapid failure. Following the general classification proposed by Brennen [2], turbopump flow instabilities can be divided in three main categories: system oscillations, global oscillations, local oscillations, and instabilities caused by radial or rotordynamic forces. Surge and cavitation auto-oscillations are system instabilities, involving strong longitudinal flow and pressure oscillations of the whole suction line, usually occurring in noncavitating pumps for positive slopes of the head characteristics (surge) and near breakdown conditions in cavitating turbopumps (cavitation auto-oscillations). Rotating stall and rotating cavitation are considered global oscillations, which propagate in the azimuthal direction at nonsynchronous 
angular speeds, typically subsynchronous for rotating stall and supersynchronous for rotating cavitation.

The first comprehensive experimental characterization of rotating cavitation instabilities was reported by Kamijo et al. [3]. They detected the occurrence of slightly supersynchronous rotating cavitation on a three-bladed inducer, leading to strong vibrations of the pump shaft at the same frequency, and suggested a simple modification of the pump housing in order to suppress this instability. In the following years, research efforts on flow instabilities increased, particularly in Japan. Hashimoto et al. [4] analyzed in detail the influence of the number of blades, carrying out experiments on 3 and 4-bladed inducers. On both inducers they detected the occurrence of cavitation surge, rotating cavitation and steady asymmetric cavitation. In addition, on the four-bladed inducer they observed a form of alternate blade cavitation, an instability similar to steady asymmetric cavitation and typical of inducers with an even number of blades, where two cavitation cells propagated synchronously at the pump rotational speed. Tsujimoto and his collaborators [5] introduced the use of crosscorrelation and phase analysis techniques in the experimental analysis of cavitation instabilities, in order to recognize oscillations of higher order characterized by a certain number of rotating cells. In the United States, Zoladz [6] carried out a series of experiments at the NASA Marshall Space Flight Center during the development of the LOX turbopump of the Fastrac engine. An aluminum prototype of the inducer of this turbopump failed due to the strong oscillating stresses caused by cavitation surge and rotating cavitation.

It is worth noticing that the occurrence of rotating cavitation has been extensively reported in the development of most high performance liquid propellant rocket fuel feed systems, including the Space Shuttle Main Engine (Ryan et al. [7]), the European Ariane 5 engine (Goirand et al. [8]) and the LE-7 engine of the H-II and H-II-A Japanese rockets (Kamijo et al. [3]). More recently, Tsujimoto and Semenov [9] identified in the LE-7 inducers a new high-order cavitation surge (axial) instability, occurring at frequencies as high as 4 to 5 times the pump rotational speed and involving a periodic redistribution of cavitation along the blade channels, with no net change of the total cavitation volume. Contrary to conventional cavitation auto-oscillations, high order cavitation surge is not therefore a system instability, as also suggested by its much higher frequency. According to the authors, its unforeseen resonance with the first bending mode of the inducer blades was responsible for the fatigue failure of the liquid hydrogen pump inducer of the 8th launch of H-II rocket in November 1999. Similar high-order instabilities, both longitudinal and rotating, have been also found on space rocket-type inducers by US researchers (Subbaraman et al., [10], [11]).

A reduced order model for the preliminary design and the prediction of the noncavitating performance of tapered axial inducers has been recently developed at Alta S.p.A. in the framework of a research program funded by the European Space Agency. The main features of the model have been extensively presented in two previous papers (d'Agostino et al., [12], [13]), and can be summarized as follows. Under the assumptions of incompressible, inviscid and irrotational flow, the model approximates the $3 \mathrm{D}$ flow field inside the blade channels by superposing a 2D cross-sectional vorticity correction to a fully-guided axisymmetric flow with radially uniform axial velocity. Suitable redefinition of the diffusion factor to bladings with non-negligible radial flow allows for integral control of the blade loading and for the estimate of the boundary layer blockage at the specified design flow coefficient, providing a simple way to better match the hub profile with the axial variation of the blade angle in variablepitch tapered inducers. Carter's rule is used to account for flow deviation at the inducer trailing edge. Mass continuity, angular momentum conservation and the Euler equation are used to propagate the solution downstream and derive a simple 2nd order ordinary differential boundary value problem, whose numerical solution yields the steady, axisymmetric, axial flow at the inducer discharge. Finally, the noncavitating pumping performance is obtained by introducing suitably adapted factors to the blade viscous losses derived from the estimated value of the flow blockage at design conditions.

The model has been preliminarily validated by comparing its results with the experimental data obtained from tests on several space rocket inducers (Cervone et al., [14], [15], [16]), including a prototype of the four-bladed inducer, manufactured in Italy by Avio S.p.A. for the LOX turbopump of the Ariane Vulcain MK1 rocket engine, and a second inducer, called FAST2, also designed by Avio S.p.A. and derived from the VINCI180 inducer. This preliminary validation has been completed by comparing the model results with the experimental performance reported in the literature for a number of inducers tested in Japanese laboratories.

One of the main features of the analytical model is the possibility of providing a useful tool for rapid geometric definition and preliminary design of space rocket axial inducers. In particular, it has been successfully used at Alta S.p.A., Pisa, Italy, for designing and manufacturing two inducers (with 3 and 4 blades, respectively), named DAPAMITO. The present paper illustrates the main results of an extensive experimental campaign conducted on the 3-bladed DAPAMITO inducer, with particular emphasis on noncavitating/cavitating performance and flow instabilities.

\section{EXPERIMENTAL APPARATUS}

The experimental activity illustrated in the present paper has been carried out in the Cavitating Pump Rotordynamic Test Facility (CPRTF, Figure 1), a low-cost, versatile and easily instrumentable facility, operating in water at temperatures up to $90{ }^{\circ} \mathrm{C}$ (Rapposelli et al. [17]). The facility is intended as a flexible apparatus that can readily be adapted to conduct experimental investigations on virtually any kind of fluid dynamic phenomena relevant to high performance turbopumps in a wide variety of alternative configurations (axial, radial or mixed flow, with or without an inducer). The CPRTF has been especially designed for the analysis of unsteady flow phenomena and rotordynamic impeller forces in scaled cavitation tests under fluid dynamic and thermal cavitation similarity conditions. It can also be configured as a small water tunnel to be used for thermal cavitation tests aimed at fundamental investigations and/or experimental validation of numerical tools and simulations. 


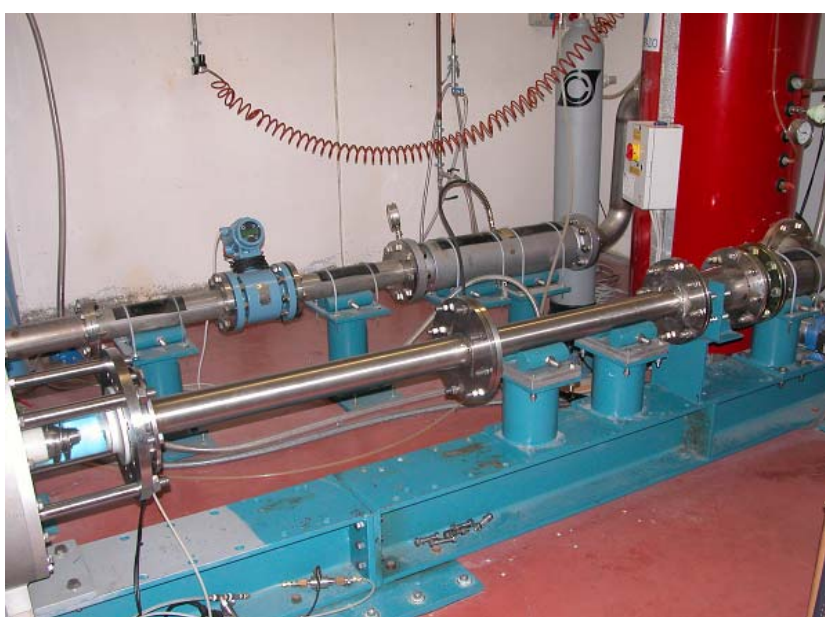

Figure 1. The Cavitating Pump Rotordynamic Test Facility.

The test section (Figure 2) is equipped with a rotating dynamometer, for the measurement of the instantaneous forces and moments acting on the impeller, and with a mechanism capable of adjusting and rotating the eccentricity of the impeller axis in the range from 0 to $2 \mathrm{~mm}$ and $\pm 3000 \mathrm{rpm}$ for rotordynamic experiments. The inlet section, made in transparent plexiglas, allows for multilateral optical access to the inducer flow. It can be instrumented with several flushmounted piezoelectric pressure transducers (PCB M112A22, $\mathrm{ICP}^{\circledR}$ voltage mode-type, $0.1 \%$ class), located at three axial stations: the flow inlet and outlet sections and the inducer blade channels (Figure 3). At each station up to eight transducers can be mounted with a given angular spacing, in order to crosscorrelate their signals for amplitude, phase and coherence analyses. As a result, waterfall plots of the power spectral density of the pressure fluctuations can be obtained as functions of the cavitation number, in order to identify the presence of flow instabilities in the flow conditions under consideration. Cross-correlation of two pressure signals from different locations allows for determining the axial or azimuthal nature of each instability and, in the second case, the number of rotating cells involved.

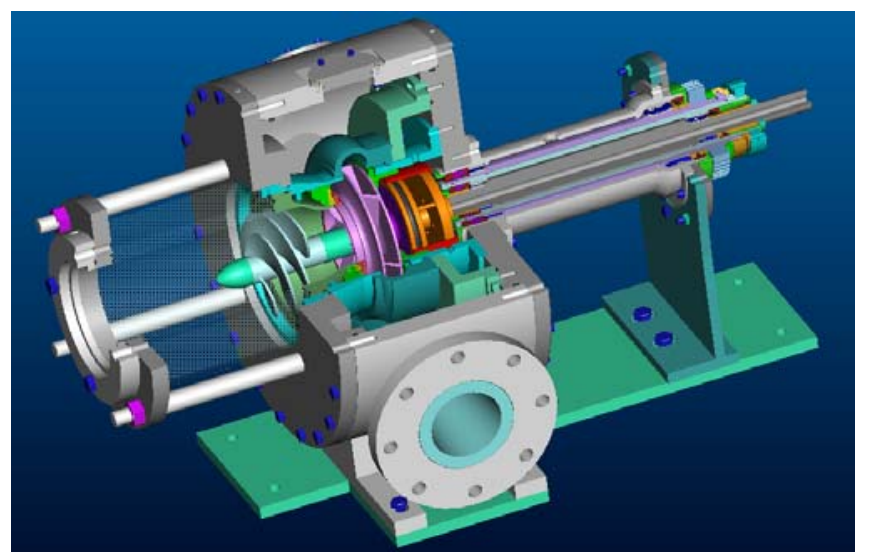

Figure 2. Cut-out drawing of the CPRTF test section.

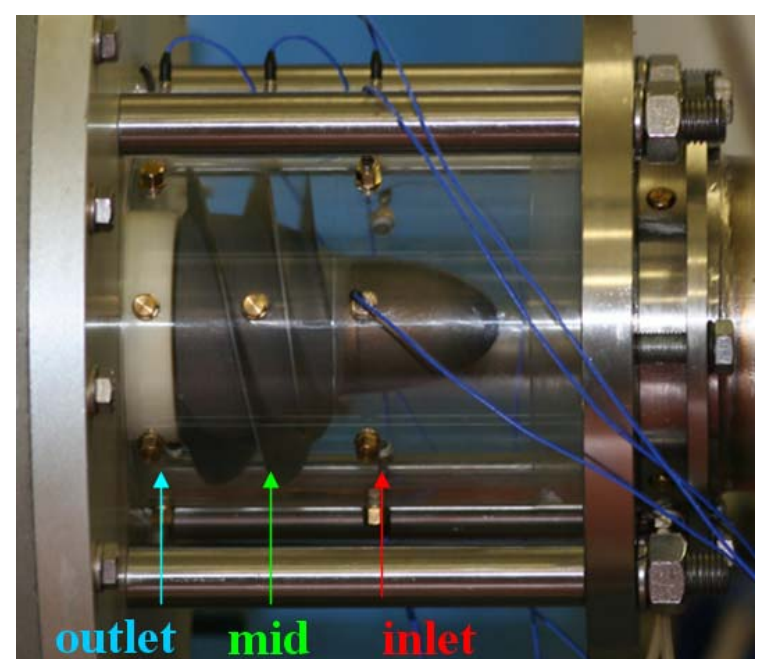

Figure 3. The transparent inlet section of the facility instrumented with piezoelectric pressure transducers.

The water pressure at the inlet of the test section can be adjusted by means of an air bag, while the temperature regulation is obtained by a $5 \mathrm{~kW}$ electrical heater. A Silent Throttle Valve is used for the variation of the pump load. Two electromagnetic flow meters (mod. $8732 \mathrm{C}$ by FisherRosemount, range $0-100 \mathrm{l} / \mathrm{s}$, accuracy $0.5 \% \mathrm{FS}$ ), mounted on the suction and discharge lines of the water loop, provide the measurement of the inlet and outlet flow rates. The inlet pressure is monitored by an absolute transducer positioned about one diameter upstream of the blade leading edges (Druck, model PMP 1400, $0 \div 1.5$ bar operating range, $0.25 \%$ precision class), while a pair of redundant differential pressure transducers measure the pump pressure rise between the same inlet section and an outlet section positioned about two diameters downstream of the blade trailing edges (Kulite, model BMD 1P 1500 100, 0 $\div 6.8$ bar-d operating range, 0.1\% precision class; Druck, model PMP 4170, 0 $\div 1$ bar-d operating range, $0.08 \%$ precision class). Photo cameras and high-speed video cameras can be used for visualization of the cavitating flow on the test article.

\section{TEST INDUCER}

As anticipated earlier, the overall dimensions of the DAPAMITO inducers have been chosen for installation and testing in the current CPRTF configuration. Typical geometries and operational characteristics of high-head space rocket inducers - and specifically the MK1 Vulcain inducer [15] have been used for reference in the design procedure. Moderate values of the blade loading and high solidities have been chosen for reducing the leading edge cavity and improving the suction performance. An incidence-to-blade angle ratio $\alpha / \beta_{b}<$ 0.5 has been selected with the aim of controlling the danger of surge instabilities at design flow under cavitating conditions. In the 3-bladed DAPAMITO inducer (used for the present experimental campaign), the tip radius is $81 \mathrm{~mm}$, the hub radius is $44.5 \mathrm{~mm}$ at the blade leading edge and $58.5 \mathrm{~mm}$ at the inducer outlet. The inlet tip blade angle is $83.1^{\circ}$ and the tip solidity is 2.03 . The nominal flow coefficient of this inducer is equal to 0.059 . 
Pictures of the inducer are presented in Figures 4 and 5 . Figure 5 , in particular, shows a frontal view of the inducer mounted in the test chamber of the facility, with the piezoelectric pressure transducers flush-mounted at a number of azimuthal locations.

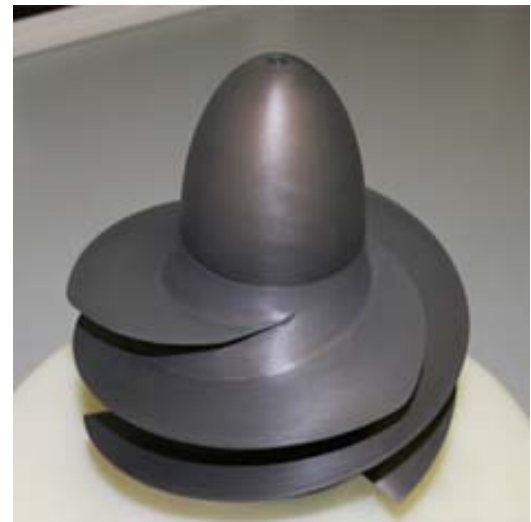

Figure 4. The 3-bladed DAPAMITO inducer.

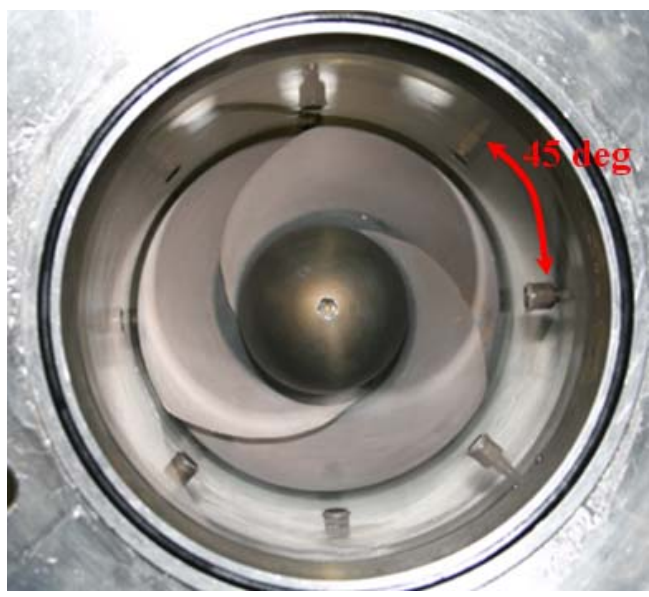

Figure 5. Frontal view of the inducer inside the facility test chamber.

\section{RESULTS AND DISCUSSION}

Noncavitating Performance

The noncavitating performance of the DAPAMITO inducer, in terms of the static head coefficient $\psi=\Delta p / \rho \Omega^{2} r_{T}^{2}$ as a function of the flow coefficient $\phi=Q / \pi \Omega r_{T}^{3}$, is shown in Figure 6.

In view of an extensive testing phase directed to the study of rotordynamic forces under eccentric motion conditions, experiments have been carried out at a high value of the blade tip clearance ( $2 \mathrm{~mm}$, equal to $6.8 \%$ of the mean blade height). This led to $34 \%$ static head loss with respect to the expected performance at nominal clearance, and to significant consequences in terms of detected flow instabilities (as shown in the following). Nevertheless, as shown in the Figure, the non-cavitating performance has been correctly predicted by the analytical model used for designing the inducer (hereby applied in a modified form, including an additional correction for taking into account blade tip clearance effects).
Experimental data are presented for three different values of the rotating speed: 1500,2000 and $2500 \mathrm{rpm}$. All of these cases refer to Reynolds numbers $R e=2 \Omega r_{T}^{2} / v$ higher than $10^{6}$, in order to attain independence of the inducer performance on turbulence effects.

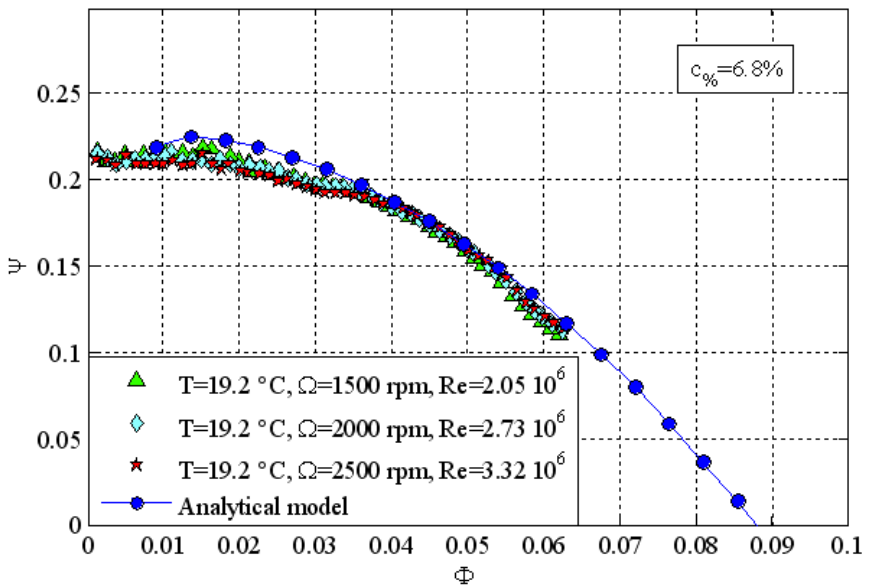

Figure 6. Noncavitating performance of the 3-bladed DAPAMITO inducer. Static head coefficient plotted as a function of the flow coefficient at room water temperature and inlet pressure for different rotating speeds, and comparison with the predictions of the analytical model.

\section{Cavitating performance}

Figure 7 shows the cavitating performance of the inducer in terms of the head coefficient as a function of the cavitation number $\sigma=\left(p_{\text {in }}-p_{V}\right) / \frac{1}{2} \rho \Omega^{2} r_{T}^{2}$ for several values of the flow coefficient. In the present case, the static pressure at inducer inlet has been used for the evaluation of the cavitation number.

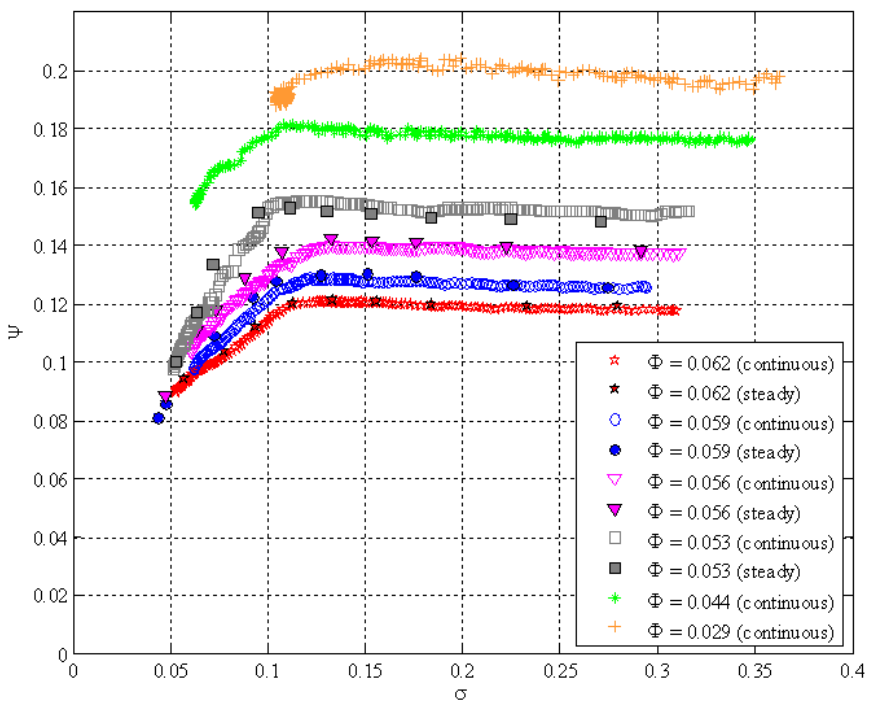

Figure 7. Cavitating performance of the DAPAMITO inducer. Static head coefficient plotted as a function of the cavitation number at room water temperature for $\Omega=3000 \mathrm{rpm}$ and several values of the flow coefficient. Comparison between "steady" and "continuous" tests is also shown. 
The plot of Figure 7 also shows a comparison between the results obtained using two different test procedures, namely "steady" and "continuous" experiments. More in detail, "steady" tests have been carried out at fixed values of the flow coefficient and cavitation number, while "continuous" tests have been conducted by keeping the flow coefficient and the pump rotational speed constant and gradually reducing the inlet pressure from atmospheric conditions to the minimum allowable value, at a constant rate of about $3 \mathrm{mbar} / \mathrm{sec}$. This technique was validated during past experiments and showed to be accurate and effective for characterizing the nature and spatial configuration of the inducer flow instabilities (Cervone et al., [14], [15], [16]).

Figure 8 illustrates the appearance of cavitation on the inducer at the nominal flow coefficient and decreasing values of the cavitation number. From Figure 7 it can be noted that the breakdown cavitation number (conventionally defined as the value corresponding to $5 \%$ head performance degradation) is $\sigma=0.09$.
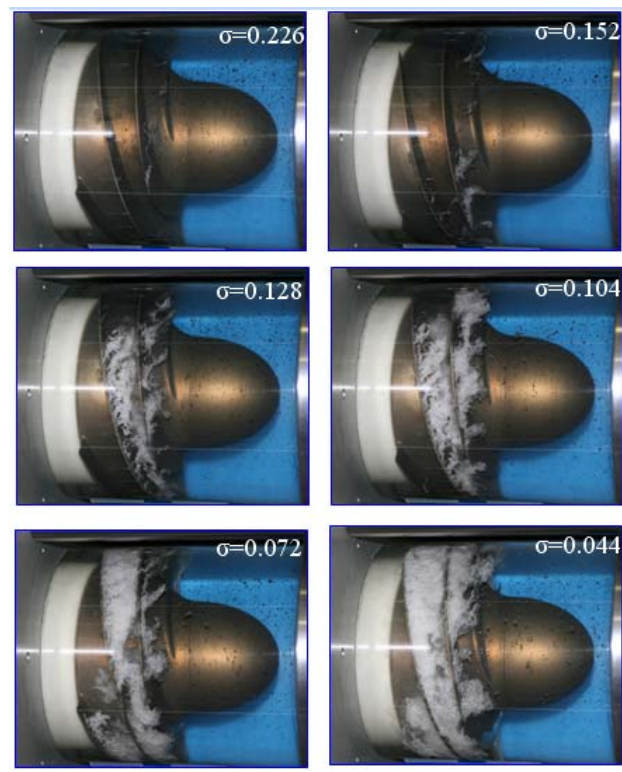

Figure 8. Cavitation appearance on the DAPAMITO inducer, at nominal flow coefficient $\phi=0.059$ and room water temperature, for $\Omega=3000 \mathrm{rpm}$ and several values of the cavitation number.

\section{Flow Instabilities}

The experiments for characterizing the inducer flow instabilities have been carried out with the same procedure used in the "continuous" tests for the evaluation of the cavitating performance. In order to characterize the occurrence and nature of rotating instabilities, six piezoelectric transducers have been mounted at the inducer inlet station (namely, $33 \mathrm{~mm}$ upstream of the leading edges of the blades) with an angular spacing of $45^{\circ}$. Pressure signals have been acquired at 1000 samples per second in order to explore potentially instable frequencies up to $500 \mathrm{~Hz}$ (equivalent to 10 times the inducer rotational speed).
A wide range of flow coefficients has been investigated, from $\phi=0.062$ down to 0.029 (respectively corresponding to $105 \%$ and $50 \%$ of the design value), but only a limited number of significant flow instabilities have been detected. This is probably due to the reduction of the blade load induced by the high value of the tip clearance, which is expected to delay the occurrence of cavitation and mitigate the intensity of the related flow instabilities.

As an example, Figure 9 shows the waterfall plot of the pressure fluctuation spectra measured at the inducer inlet section for the highest value of the flow coefficient ( $\phi=0.062$ ).

The only evident peaks correspond to the blade passing frequency $3 \Omega$ and its submultiples. The detection of the rotating frequency $\Omega$ is due to the uneven behavior of the flow on the three blades, and therefore to the presence of steady asymmetric cavitation. This finding has been confirmed by optical visualization, which demonstrated the occurrence of cavitation with different shape and size on the three blades.

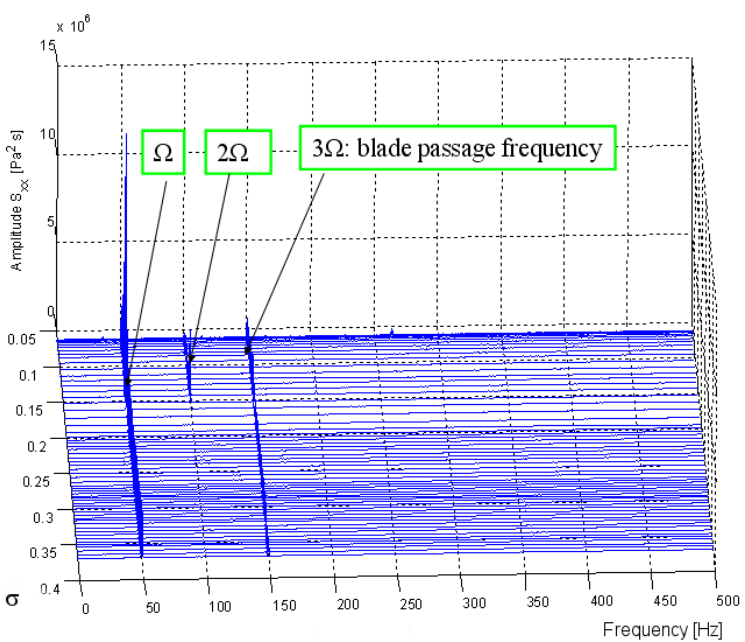

Figure 9. Waterfall plot of the power spectrum of the inlet pressure fluctuations in the DAPAMITO inducer at $\phi=0.062$, $3000 \mathrm{rpm}$ and room water temperature.

A more detailed analysis of the waterfall plot presented in Figure 9 has been carried out by filtering the contributions at $\Omega, 2 \Omega$ and $3 \Omega$ and zooming the vertical scale. This procedure has allowed for the detection of a number of weaker instabilities, as illustrated in Figure 10. These instabilities (or at least the most significant of them) are expected to become more evident in inducer configurations with lower tip clearance, as the authors intend to verify in further tests planned in the near future.

Two of the observed instabilities have been demonstrated to be 0 -th order (axial) phenomena. In particular:

- The instability occurring at $8 \div 9 \mathrm{~Hz}(0.16 \Omega \div 0.18 \Omega)$ probably corresponds to auto-oscillations of the inlet line flow excited at the natural frequency of the facility in the presence of cavitation. This phenomenon has been observed at values of the flow coefficient between $90 \%$ and $105 \%$ of the design conditions, and for a cavitation number between $90 \%$ and $120 \%$ of the breakdown value. The axial nature of 
this instability can be easily inferred from examination of Figure 11, which illustrates the amplitude and phase of the cross-spectral density and the coherence function of the pressure signals from two transducers mounted with $45^{\circ}$ angular separation in the azimuthal direction for inducer operation at $\sigma=0.12$. In this case, the cross-correlation of the pressure signals has $0^{\circ}$ phase angle, with a value of the coherence function practically equal to 1 . The same result is confirmed by the analysis of Figure 12, where the phases of the cross-correlation between couples of pressure transducers with different angular spacings are presented.

- The instability occurring at $356 \div 360 \mathrm{~Hz}(7.12 \Omega \div 7.2 \Omega)$ has been observed at higher flow coefficients $(105 \%$ of the nominal value) and tends to disappear at lower flow coefficients. It has been detected at relatively high values of the cavitation number (between $250 \%$ and $370 \%$ of the breakdown value) and appears to have the same characteristics of the "high-order longitudinal instability" already observed at Alta S.p.A. during experiments on a 2bladed axial inducer (Cervone et al. [16]).

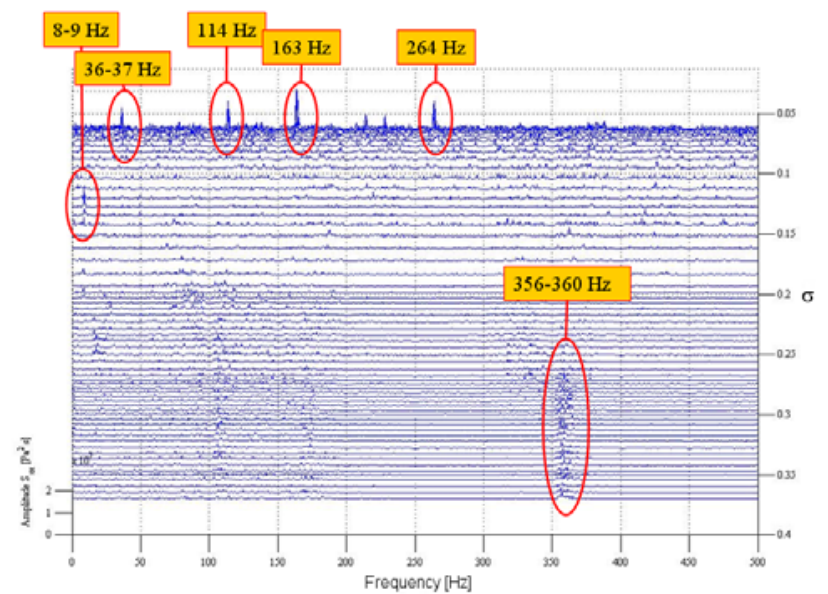

Figure 10. Filtered waterfall plot of the power spectrum of the inlet pressure fluctuations in the DAPAMITO inducer at $\phi=0.062, \Omega=3000 \mathrm{rpm}$ and room water temperature.
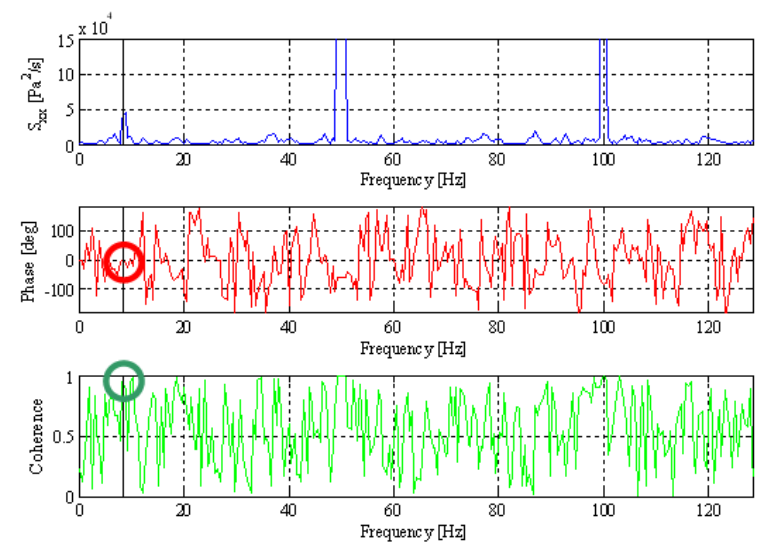

Figure 11. Amplitude and phase of the cross-spectral density and coherence function of the pressure signals of two transducers with $45^{\circ}$ angular separation mounted at the inlet section of the DAPAMITO inducer ( $\phi=0.062, \sigma=0.12, \Omega=$ $3000 \mathrm{rpm})$.

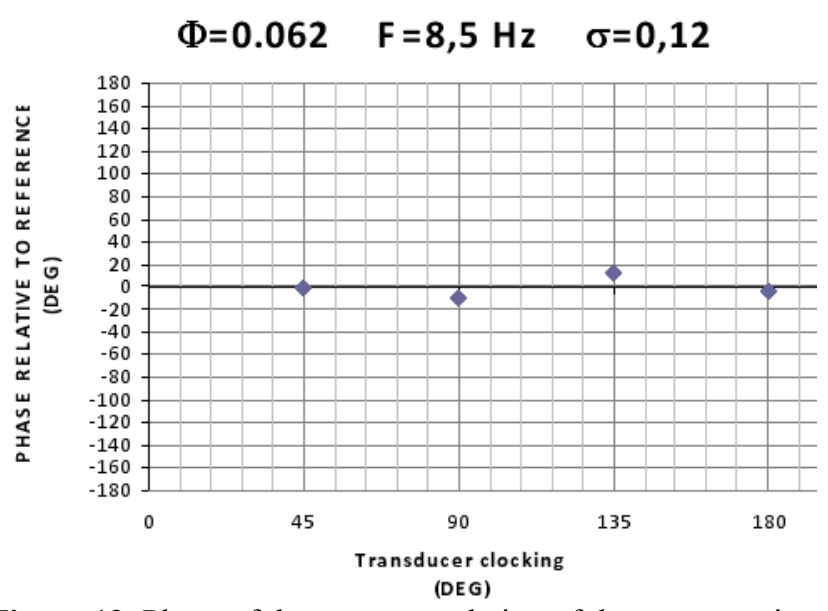

Figure 12. Phase of the cross-correlation of the pressure signals from pairs of transducers mounted with different angular separations in the inlet section of the DAPAMITO inducer ( $\phi=0.062, \sigma=0.12, \Omega=3000 \mathrm{rpm}, F=8.5 \mathrm{~Hz}$ ).

The other instabilities shown in Figure 10 are rotating phenomena, and their interpretation is significantly more difficult. All of these rotating phenomena have been detected at virtually all values of the flow coefficient, and at significantly low values of the cavitation number $(60 \%$ of the breakdown value or less). More in detail:

- The phenomenon at $36 \div 37 \mathrm{~Hz}(0.72 \Omega \div 0.74 \Omega)$ is a 4-cells rotating instability, as it can be inferred by the phase/coherence diagram of Figure 13 and by the plot of Figure 14 (phase of the cross-correlation between couples of pressure transducers at different angular spacings).

- The phenomenon at $114 \mathrm{~Hz}(2.28 \Omega)$ is a single-cell rotating instability.

- The phenomenon at $163 \mathrm{~Hz}(3.26 \Omega)$ is a single-cell rotating instability.

- The phenomenon at $264 \mathrm{~Hz}(5.28 \Omega)$ is a 2-cells rotating instability, as clearly shown by the cross-correlation/phase analysis presented in Figure 15.

\section{CONCLUSIONS}

An experimental campaign has been carried out in the Cavitating Pump Rotordynamic Test Facility at Alta S.p.A. for the characterization of the DAPAMITO inducer, a three-bladed axial pump designed and manufactured by Alta S.p.A. using a simplified analytical model for the prediction of geometry and noncavitating performance of typical space rocket inducers.

The main conclusions drawn from the experimental activity can be summarized as follows:

- The experiments have been carried out at high blade tip clearance $(6.8 \%$ of the mean blade height). As a consequence, a $34 \%$ static head loss has been observed with respect to the expected performance at nominal clearance. Additional tests at lower values of the tip clearance are foreseen in the next future in order to confirm this finding and further investigate the phenomenon. 

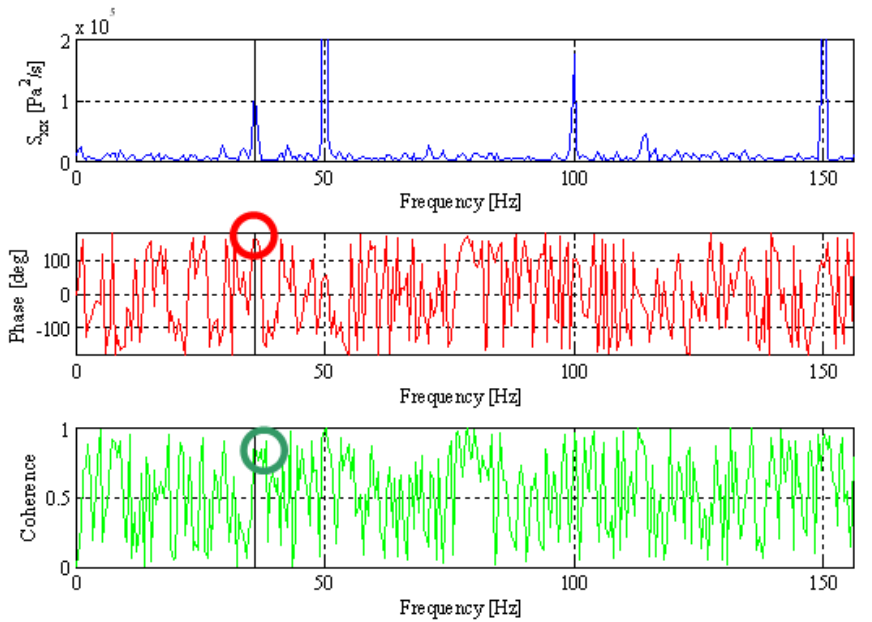

Figure 13. Power density spectrum, phase of the crosscorrelation and coherence function of the pressure signals of two transducers with $45^{\circ}$ angular separation in the inlet section of the DAPAMITO inducer ( $\phi=0.062, \sigma=0.062, \Omega=3000$ rpm).

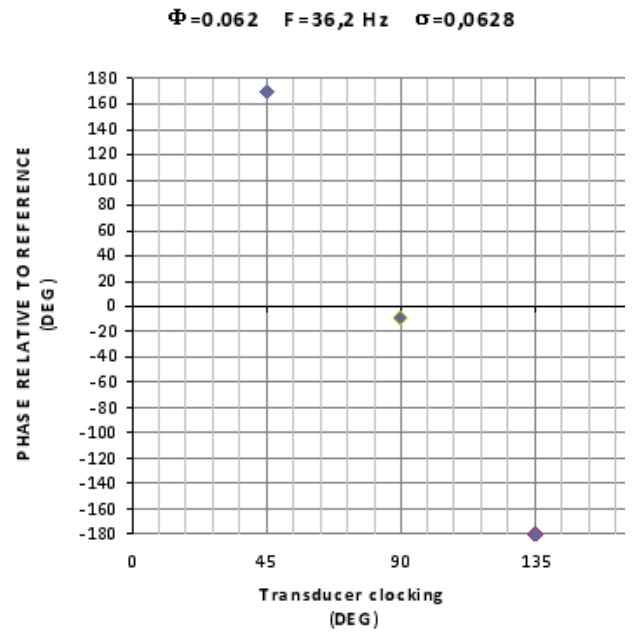

Figure 14. Phase of the cross-correlation of the pressure signals of transducers at different angular separation in the inlet section of the DAPAMITO inducer $(\phi=0.062, \sigma=0.062, \Omega=3000$ rpm, $F=36.2 \mathrm{~Hz}$ ).

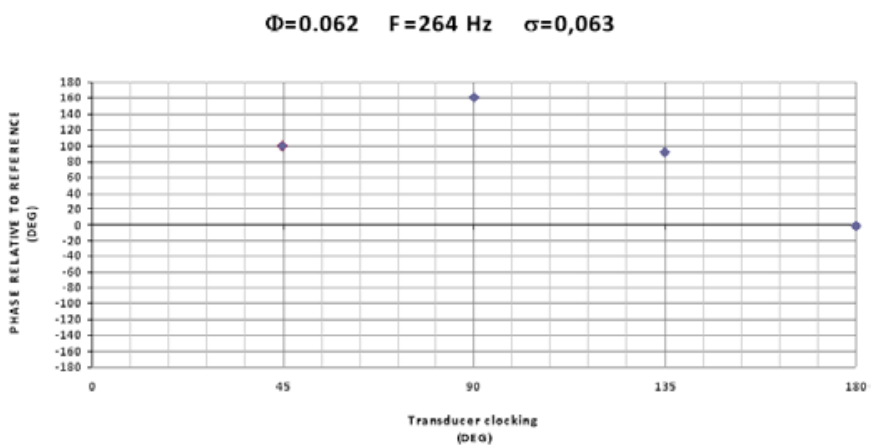

Figure 15. Phase of the cross-correlation of the pressure signals of transducers at different angular separation in the inlet section of the DAPAMITO inducer ( $\phi=0.062, \sigma=0.063, \Omega=3000$ rpm, $F=264 \mathrm{~Hz}$ ).
- A wide range of flow coefficients have been investigated between $50 \%$ and $105 \%$ of the design value, but a limited number of significant flow instabilities have detected, probably as a consequence of the high value of blade tip clearance.

- Steady asymmetric cavitation has been identified on the inducer by both the analysis of inlet pressure cross-spectra and optical visualization of inducer blade cavitation.

- Other instabilities detected in the inducer include: a) cavitation surge oscillations at a frequency equal to $0.16 \Omega \div 0.18 \Omega$ occurring at flow coefficients between $90 \%$ and $105 \%$ of the design value and cavitation numbers from $90 \%$ to $120 \%$ of the breakdown value; b) a high-order axial instability at a frequency of $7.12 \Omega \div 7.2 \Omega$ observed at higher values of the flow coefficients (105\% of the design value).

- Some rotating phenomena have also been observed on the inducer, but their interpretation is significantly more difficult. All of these rotating phenomena have been detected at virtually all values of the flow coefficient and at significantly low values of the cavitation number $(60 \%$ of the breakdown value, or lower).

\section{ACKNOWLEDGMENTS}

The research activity presented in this paper, as well as the realization of the CPRTF, have been supported and funded under European Space Agency and Italian Space Agency contracts. The authors would like to express their gratitude to Profs. Mariano Andrenucci, Renzo Lazzeretti and Fabrizio Paganucci of the Dipartimento di Ingegneria Aerospaziale, Università di Pisa, Italy, for their constant and friendly encouragement. A special acknowledgement goes to the students and colleagues who have supported the research group throughout the years with their precious help and contributions. A very special thank to Dr. K. Shinkatsu for his invaluable and constant support.

\section{NOMENCLATURE}

$\begin{array}{ll}f, F & =\text { frequency } \\ p_{i n} & =\text { inlet static pressure } \\ p_{V} & =\text { vapor pressure } \\ Q & =\text { volumetric flow rate } \\ r_{T} & =\text { inducer tip blade radius } \\ R e & =\text { Reynolds number } \\ T & =\text { working fluid temperature } \\ \Delta p & =\text { inducer static pressure rise } \\ \alpha & =\text { tip incidence angle } \\ \beta_{b} & =\text { tip blade angle } \\ \phi & =\text { flow coefficient } \\ \psi & =\text { head coefficient } \\ \rho & =\text { density } \\ \sigma & =\text { cavitation number } \\ v & =\text { kinematic viscosity } \\ \Omega & =\text { rotational speed }\end{array}$




\section{REFERENCES}

[1] Stripling L.B. and Acosta A.J., "Cavitation in Turbopumps - Part 1", ASME J. Basic Eng., Vol. 84, pp. 326-338, 1962.

[2] Brennen C.E., "Hydrodynamics of Pumps", Concepts ETI, Inc. and Oxford University Press, 1994.

[3] Kamijo K., Yoshida M. \& Tsujimoto Y., "Hydraulic and Mechanical Performance of LE-7 LOX Pump Inducer", J. Propulsion and Power, Vol. 9, No. 6, pp. 819-826, 1993.

[4] Hashimoto T., Yoshida M., Watanabe M., Kamijo K., Tsujimoto Y., "Experimental Study of Rotating Cavitation of Rocket Propellant Pump Inducers", J. Propulsion and Power, Vol. 13, N. 4., pp. 488-494, 1997.

[5] Tsujimoto Y., Yoshida Y., Maekawa Y., Watanabe S., Hashimoto T., "Observation of Oscillating Cavitation of an Inducers", ASME J. Fluids Eng. ing, Vol. 119, pp. 775-781, 1997.

[6] Zoladz T., "Observations on Rotating Cavitation and Cavitation Surge from the Development of the Fastrac Engine Turbopump", 36th AIAA/ASME/SAE/ASEE Joint Propulsion Conference, Huntsville, AL, USA, 2000.

[7] Ryan R.S., Gross L.A., Mills D., Michell P., "The Space Shuttle Main Engine Liquid Oxygen Pump HighSynchronous Vibration Issue, the Problem, the Resolution Approach, the Solution", 30th AIAA/ASME/SAE/ASEE Joint Propulsion Conference, Indianapolis, USA, 1994.

[8] Goirand B., Mertz A.L., Jousselin F., Rebattet C., "Experimental Investigations of Radial Loads Induced by Partial Cavitation with Liquid Hydrogen Inducer", IMechE, C453/056, pp. 263-269, 1992.

[9] Tsujimoto Y., Semenov Y. A., "New Types of Cavitation Instabilities in Inducers", 4th Int. Conf. on Launcher Technology, Liege, Belgium, 2002.

[10] Subbaraman M. and Burton K., "Cavitation-Induced Vibrations in Turbomachinery: Water Model Exploration", CAV2003, $5^{\text {th }}$ Int. Symposium on Cavitation, Osaka, Japan, 2003.
[11] Subbaraman M. and Patton M., "Suppressing HigherOrder Cavitation Phenomena in Axial Inducers", CAV2006, $6^{\text {th }}$ Int. Symposium on Cavitation, Wageningen, The Netherlands, 2006.

[12] d'Agostino L., Torre L., Pasini A., Cervone A., "On the Preliminary Design and Noncavitating Performance of Tapered Axial Inducers", ASME Journal of Fluids Engineering, Vol. 130, Is. 11, November 2008

[13] d'Agostino L., Torre L., Pasini A., Baccarella D., Cervone A., Milani A., "A Reduced Order Model for Preliminary Design and Performance Prediction of Tapered Inducers: Comparison with Numerical Simulations", 44th AIAA/ASME/SAE/ASEE Joint Propulsion Conference, Hartford, USA, 2008.

[14] Cervone A., Torre L., Fotino D., Bramanti C., d'Agostino L., "Characterization of Cavitation Instabilities in Axial Inducers by Means of High-Speed Movies", 42nd AIAA/ASME/SAE/ASEE Joint Propulsion Conference, Sacramento, USA, 2006.

[15] Cervone A., Testa R., Bramanti C., Rapposelli E. and d'Agostino L., "Thermal Effects on Cavitation Instabilities in Helical Inducers", AIAA Journal of Propulsion and Power, Vol. 21, No. 5, pp. 893-899, SepOct 2005.

[16] Cervone A., Torre L., Bramanti C., Rapposelli E. and d'Agostino L., "Experimental Characterization of Cavitation Instabilities in the Avio FAST2 Inducer", 41st AIAA/ASME/SAE/ASEE Joint Propulsion Conference, Tucson, USA, 2005.

[17] Rapposelli E., Cervone A., d'Agostino L., "A New Cavitating Pump Rotordynamic Test Facility", 38th AIAA/ASME/SAE/ASEE Joint Propulsion Conference, Indianapolis, USA, 2002. 